\title{
Underwater Acoustic Communication Quality Evaluation Model Based on USV
}

\author{
Zhichao Lv, ${ }^{1,2}$ Jie Zhang $\mathbb{D}^{3}{ }^{3}$ Jiucai Jin $\mathbb{D}^{1},{ }^{3}$ Qi Li, ${ }^{1,2}$ Lanjun Liu, ${ }^{4}$ \\ Pengcheng Zhang, ${ }^{4}$ and Baoru Gao ${ }^{4}$ \\ ${ }^{1}$ Acoustic Science and Technology Laboratory, Harbin Engineering University, Harbin 150001, China \\ ${ }^{2}$ College of Underwater Acoustic Engineering, Harbin Engineering University, Harbin 150001, China \\ ${ }^{3}$ The First Institute of Oceanography, State Oceanic Administration, Qingdao 266100, China \\ ${ }^{4}$ College of Engineering, Ocean University of China, Qingdao 266100, China
}

Correspondence should be addressed to Jiucai Jin; jinjiucai@fio.org.cn

Received 29 July 2017; Accepted 27 December 2017; Published 18 March 2018

Academic Editor: Mario Terzo

Copyright (C) 2018 Zhichao Lv et al. This is an open access article distributed under the Creative Commons Attribution License, which permits unrestricted use, distribution, and reproduction in any medium, provided the original work is properly cited.

The unmanned surface vehicle (USV) integrated with acoustic modems has some advantages such as easy integration, rapid placement, and low cost, which becomes a kind of selective novel node in the underwater acoustic (UWA) communication network and a kind of underwater or overwater communication relay as well. However, it is difficult to ensure the communication quality among the nodes on the network due to the random underwater acoustic channel, the severe marine environment, and the complex mobile node system. Aiming to model the communication characteristics of the USV, the multipath effect and Doppler effect are main concerns for the UWA communication in this paper, so that the ray beam method is utilized, the channel transmission function and the channel gain are obtained, and the mobile communication quality evaluation model is built. The simulation and lake experiments verify that the built mobile UWA communication quality evaluation model on USV can provide preference and technique support for USV applications.

\section{Introduction}

As unmanned platform and relative technology developed, the unmanned platform has become a hotspot for the ocean technique research and development which provides technique support for underwater network mobile relay and topology optimization [1]. In particular, the USV integrated with underwater acoustic modem has some advantages such as easy integration, rapid placement, and low cost so that it becomes a selective novel node for underwater network and a kind of underwater or overwater communication relay as well. Researchers at the University of Porto used the unmanned ship called ZARCO to carry out the communication and positioning research among the autonomous underwater vehicles (AUVs) [2]. Massachusetts Institute of Technology researched and developed the unmanned ship SCOUT [3]. They realized the underwater communication among several USV by UWA communication modems, which can be used for developing the unmanned ship based communication and positioning system. Murphy and his fellows developed an underwater communication system CAPTURE and designed the UWAC abstract physical layer for providing underwater data and image communication relay of different resolution ratio between mother vessel and AUVs [4]. Several recycle experiments including underwater data and sonar image were carried out in 2011 and 2012, which verified the practicability of the system. However, all these mentioned application experiments have met the same problem that UWA channel is time-space-frequency varying. In this experiment, the narrow pass band, the strong multipath effect, the severe signal fading, and the obvious Doppler effect have caused the instability of the communication link [5]. Currently, underwater acoustic communications still rely on extensive field experiments to evaluate the performance of the physical-layer algorithms [6].

In recent years, many efforts have been done to characterize the path statistics which showed that some simple channels could be used to describe the communication 
link. These statistic models included the Rayleigh [7], the Rician [8], the Nakagami-m [9], and the $K$ distributed probability distribution [10]. It was not considered that the parameters affect the communication performance for the UWA channel model. For simplification, some researchers used simple method for communication ability estimation such as calculating the packet loss probability which relied on the communication distance only [11]. In addition, the surface of the rolling model was introduced into the hybrid model, which was used for simulating the surface influence precisely on signal Doppler frequency shift, analyzing the channel correlation function characteristics and secondorder statistical properties [12-15]. Part of the problem is that there is not a standard underwater acoustic channel model by now. The stochastic reproduction of the model has not been successfully compared with the real data, except for the data whose statistic is known [6].

Therefore, we propose to build the UWA communication quality evaluation model based on the USV (USVUWACQE) in this paper. The researches of communication quality of the UWA communication network were relatively rare, especially the situation which applied to the UWA communication model of the USV. The USV-UWACQE model is considered for the first time, mainly for the multipath effect and Doppler effect, the ray beam method is utilized, the channel transmission function and the channel gain are obtained, and the mobile communication quality evaluation model is built.

The remainder of the paper is structured as follows. Section 2 describes the USV-UWACQE model from the influence of multipath and Doppler effect. Section 3 conducts simulation and lake experiments to verify and analyze our models. Section 4 concludes our work and discusses some future work.

\section{Model Analysis}

Due to the background for the special application of mobile underwater acoustic communication based on the USV, the multipath effect and Doppler effect have a greater impact on the communication quality. Therefore, we conduct the underwater acoustic communication quality evaluation model based on the unmanned surface vehicle (USV-UWACQE) by combining the characteristic of the mobile USV itself and analyzing the impact factor, such as multipath effect, Doppler effect factor, and acoustic signal absorption and expansion. First, we obtain the channel transfer function with the ray beam method $[12,13]$ :

$$
H(f)=H_{0}(f) \sum_{n} h_{n} e^{-j 2 \pi\left(a_{n}+1\right) f \tau_{n}},
$$

where $H_{0}$ is the channel transfer function of the first path signal, $f$ is the natural frequency of the signal, $h_{n}$ is the impulse response function of the $n$th path, $a_{n}$ is the Doppler factor of the $n$th path, and $\tau_{n}$ is the delay of the $n$th path.

The integer of the channel transfer function in frequency bandwidth can be obtained by

$$
G=\frac{1}{B} \int_{f_{0}}^{f_{0}+B}|H(f)|^{2} d f
$$

where $B$ represents the bandwidth of underwater acoustic communication signal, $f_{0}$ is the initial frequency, and $G$ is the channel gain.

$$
S_{R}=S_{T}-G,
$$

where $S_{R}$ is the strength of the received signal, which is the evaluation metrics of the USV-UWACEQ model. $S_{T}$ is the strength of the launched signal.

2.1. Influence of Multipath in the Model. For underwater acoustic communication, seawater is a kind of nonideal loss medium. The signal intensity will decline when transmitted in the medium. In the model of underwater acoustic communication link quality, the decline of this signal can cause energy loss. We consider that the link's energy loss is mainly caused by the wave front expansion, the assimilation of the seafloor, the sea surface, and the sea medium. By ignoring specific propagation, the acoustic signal loss can be estimated by the empirical formula as follows [8]:

$$
A(l, f)=\left(\frac{l}{l_{r}}\right)^{k} a(f)^{l-l_{r}},
$$

where $A(l, f)$ is the absorption expansion loss, $f$ is the natural frequency of the signal, $a$ is the absorption coefficient, and $l$ is the distance.

Because of the random scattering of medium and the reflections of the seafloor, the sea surface, and the obstructions in the propagation of the sound waves, the sound signal cannot reach the hydrophone at the same time, which leads to the multipath effect of the underwater acoustic channel.

The influence of multipath is very obvious in the underwater acoustic communication system. When the guard interval of each symbol is less than that of multipath delay, the signal symbol overlaps at the time of receipt bringing in the intersymbol interface and increasing the bit error rate. It is assumed that a signal propagates between the transducer and the hydrophone which generates $N$-path signal. And because the relative position of the node is constantly changing, the distance and the propagation time of each path signal is different. We assume that the propagation distance of each path is $l_{n}(n=0, \ldots, N-1)$ and the transfer function is defined as

$$
H_{n}(f)=\frac{\Gamma_{n}}{\sqrt{A\left(l_{n}, f\right)}},
$$

where $\Gamma_{n}$ indicates the total reflection coefficient of the $n$-path sound signal which is reflected by the seafloor and the sea surface. And $A\left(l_{n}, f\right)$ is the absorption expansion loss which is represented in (4).

Hence, the transfer function of the communication system can be calculated by

$$
H(f)=\sum_{n} H_{n}(f) e^{-j 2 \pi f \tau_{n}},
$$

where $\tau_{n}$ is the propagation delay of the $n$-path signal which can be obtained by $\tau_{n}=l_{n} / c-t_{0}, t_{0}=l_{0} / c$, and $c$ indicates the constant speed of the sound. 

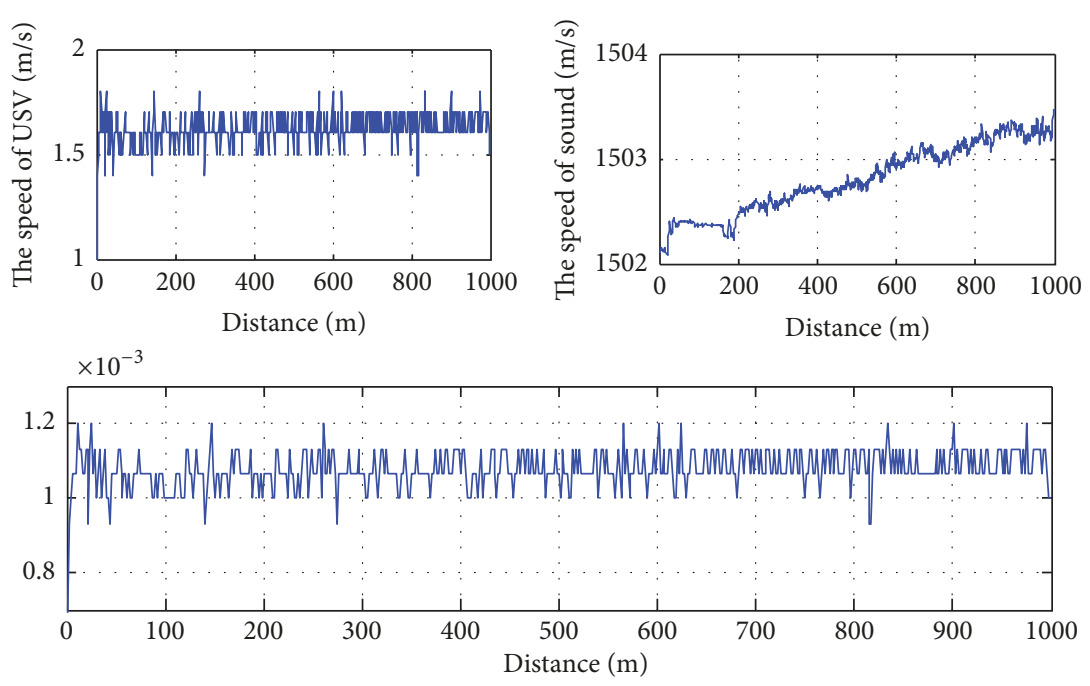

FIGURE 1: USV speed/sound speed/Doppler factor.

Due to the high frequency used in the underwater acoustic communication, the absorption coefficient approximately equals one, namely, $a(f) \approx 1$, and the transfer function can be approximated by

$$
H_{n}(f)=h_{n} H_{0}(f),
$$

where $h_{n}$ can be represented by

$$
h_{n}=\frac{\Gamma_{n}}{\sqrt{\left(l_{n} / l_{0}\right)^{k} a_{0}^{l_{n}-l_{0}}}},
$$

where $\Gamma_{n}$ indicates the total reflection coefficient of the $n$-path sound signal which is reflected by the seafloor and the sea surface. $k$ is the spreading factor.

The signal transfer function can be calculated by substituting (7) into (6):

$$
H(f)=H_{0}(f) \sum_{n} h_{n} e^{-j 2 \pi f \tau_{n}}
$$

where $H_{0}$ is the channel transfer function of the first path signal, $f$ is the natural frequency of the signal, $h_{n}$ is the impulse response function of the $n$th path, and $\tau_{n}$ is the delay of the $n$th path.

2.2. Influence of Doppler Effect in the Model. The Doppler effect is the frequency's shift and extension caused by the relative movement between the transducer and the receiving hydrophone. The Doppler effect is described by the Doppler factor $a$ which is calculated by

$$
a=\frac{v}{c}
$$

where $v$ is the relative speed between the transducer and receiving hydrophone and $c$ is the sound speed.

We assume that the relative speed between the transmitting and receiving platforms is $1.5 \mathrm{~m} / \mathrm{s}$, the system operating frequency $f_{c}$ is $20 \mathrm{kHz}$, and the Doppler shift caused by the relative motion $f_{d}=f_{c} \cdot v / c$ is $20 \mathrm{~Hz}$. And the receiving end of the synchronization and the decoding is seriously affected in the later part, thus affecting the communication quality.

The speed of the sound has an effect on the Doppler factor. The temperature, salinity, and depth are the main parameters that influence sound speed. We can conclude the empirical formula through many measurements on the sea as follows [16]:

$$
\begin{aligned}
c= & 1449.2+4.6 T-0.555 T^{2}+0.00029 T^{3} \\
& +(1.34-0.01 T)(S-35)+0.016 z,
\end{aligned}
$$

where $T$ is the temperature, $S$ is the salinity, and $z$ is the depth.

In the communication system of the USV equipped with underwater acoustic modem, there is CTD on the USV which can collect the marine environment parameters in real time. Using the empirical formula, we can get the sound speed $c$ which can provide data support for the Doppler effect estimation and communication channel model.

Figure 1 shows that the speed of the USV, the speed of the sound, and the Doppler factor change with the distance in the USV mobile communication experiment within $1 \mathrm{~km}$. As can be seen in the figure, Doppler effect cannot be ignored in underwater acoustic communication by USV.

The vertical movement was caused by the sea surface waves and the horizontal movement was brought about by the speed of the USV affecting the Doppler factor. The speed of the USV can reach a few meters per second, and the Doppler effect on the model cannot be ignored with the influence of the wind, wave, and motion of the USV.

It is assumed that the direction of the transmitting transducer is fixed and known in the USV-UWACEQ model. And the Doppler factor can be defined by

$$
a=\frac{1}{c}\left[v_{s} \cos \left(\theta_{1}\right)+v_{v} \cos \left(\theta_{2}\right)\right],
$$

where $\theta_{1}$ is the vertical angle between the transmitting transducer and the receiving hydrophone, $\theta_{2}$ is the horizontal 


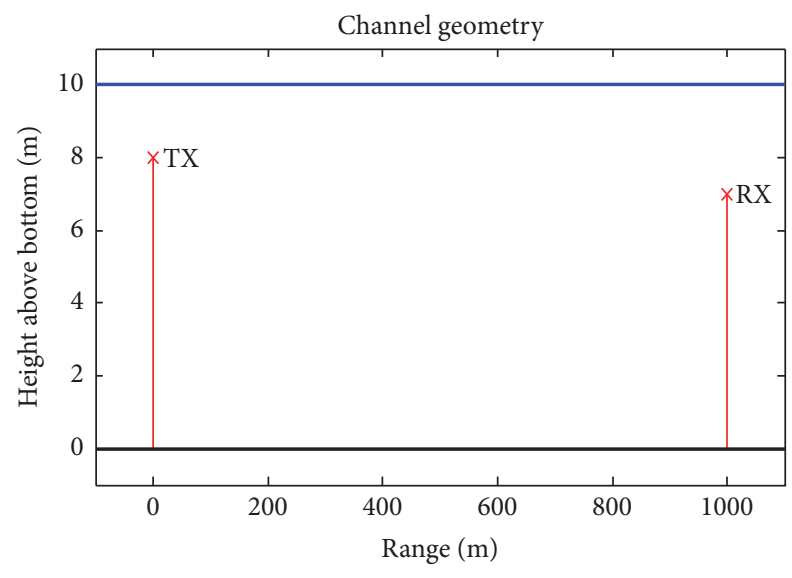

FIgURE 2: Model simulation diagram.

angle between the transmitting transducer and the receiving hydrophone, and $v_{s}$ and $v_{v}$ are the velocity of the vertical and the horizontal. In order to calculate the Doppler factor, the navigation state model of the USV is introduced, which is based on the six-degree-freedom nonlinear equations considering the impact of the marine environment, such as wind, waves, stream, and damp [17].

In the process of underwater acoustic communication, the USV motion is decomposed in horizontal and vertical parts. The USV is equipped with a Gyro attitude sensor and GPS so as to estimate the parameters, which are used to analyze the communication quality. Thus, we can draw a conclusion that these parameters mainly have an influence on the model, such as pitch rate $\dot{\theta}$ in the vertical direction, roll angular velocity $\dot{\phi}$, and velocity $v$ within horizontal plane. The $v_{s}, v_{v}, \theta_{1}$, and $\theta_{2}$ can be calculated in real time which can be used for the calculation of the Doppler factor. The Doppler factor $a$ affects each path signal in the underwater acoustic communication which can be represented by

$$
a_{n}=\frac{1}{c}\left[v_{s n} \cos \left(\theta_{1}\right)+v_{v n} \cos \left(\theta_{2}\right)\right] .
$$

The effect of the Doppler factor is reflected in the transfer function which can be obtained by

$$
H(f)=H_{0}(f) \sum_{n} h_{n} e^{-j 2 \pi\left(a_{n}+1\right) f \tau_{n}} .
$$

\section{The Simulation and Experimental Verification}

3.1. Simulation Results. We test the USV-UWACQE model on the condition of the laboratory simulation in Figure 2. The same parameters are used in the simulations and the lake experiments which are given in Table 1 .

In simulation experiments, we design the desired trajectory in a straight line according to Table 1 . The fixed and mobile simulation results are, respectively, given in Figures 3 and 4 . The results are done five times in the specified position. The horizontal axis is the distance from the transmitter to
TABLE 1: Model parameters in simulations.

\begin{tabular}{lc}
\hline Parameters & Values \\
\hline Water depth & $10 \mathrm{~m}$ \\
Depth of the transmitter & $2 \mathrm{~m}$ \\
Bandwidth & $18-34 \mathrm{kHz}$ \\
Speed of transmitting transducer & $0 \mathrm{~m} / \mathrm{s}$ \\
The distance between the transmitter and receiver & $1000 \mathrm{~m}$ \\
Depth of the receiver & $3 \mathrm{~m}$ \\
Time duration & $90 \mathrm{~s}$ \\
Speed of the receiving transducer (USV) & $2 \mathrm{~m} / \mathrm{s}$ \\
\hline
\end{tabular}

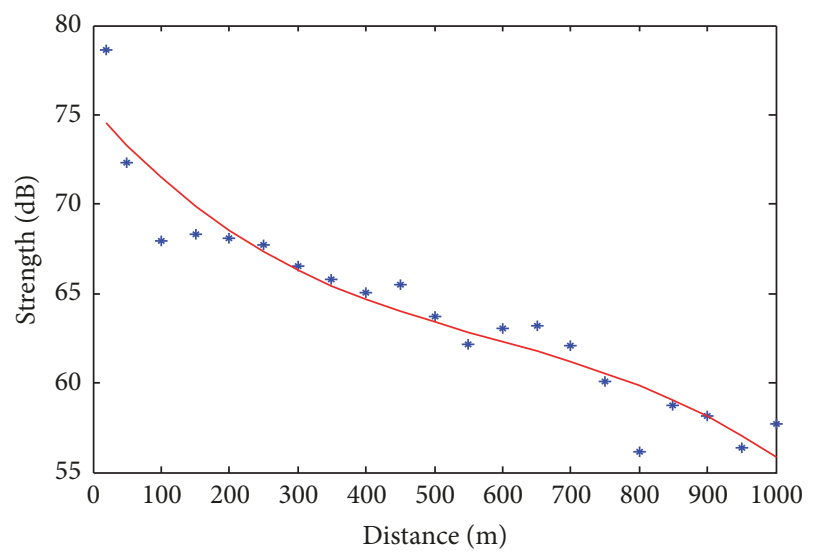

Figure 3: Fixed simulation results.

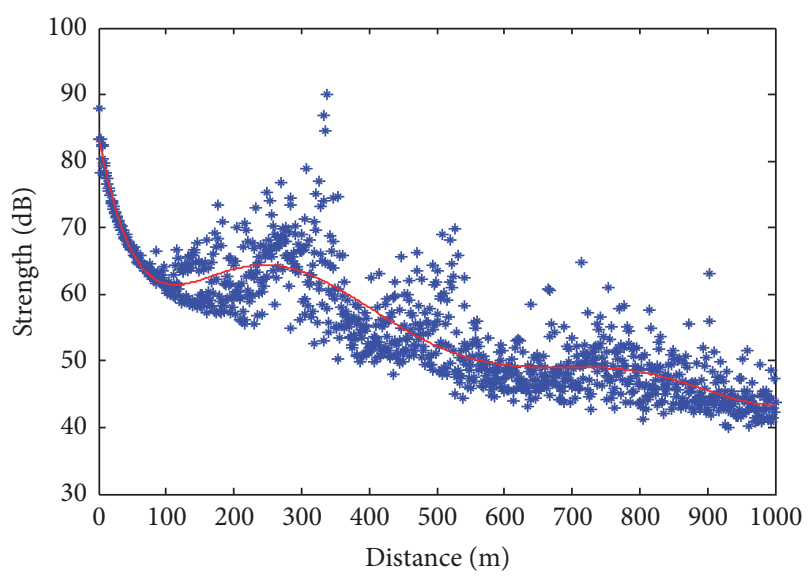

FIGURE 4: Mobile simulation results.

the receiver and the vertical axis is the signal strength. The blue asterisk indicates the average value of five results every $50 \mathrm{~m}$ and the red smooth curve represents the fitted curve in Figure 3. It can be seen that the fixed signal strength attenuates from about $80 \mathrm{~dB}$ to about $55 \mathrm{~dB}$. Similarly, the blue asterisk represents the average value of five results every one meter and the red curve also represents the fitted curve in Figure 4 . And Figure 4 shows that the mobile signal strength attenuates from about $80 \mathrm{~dB}$ to about $45 \mathrm{~dB}$. Compared with the two fitting curves, the difference between the fixed and 


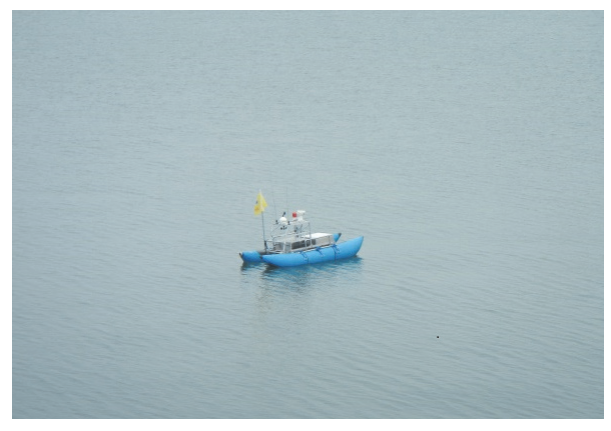

FIGURE 5: Our USV in the lake experiment.
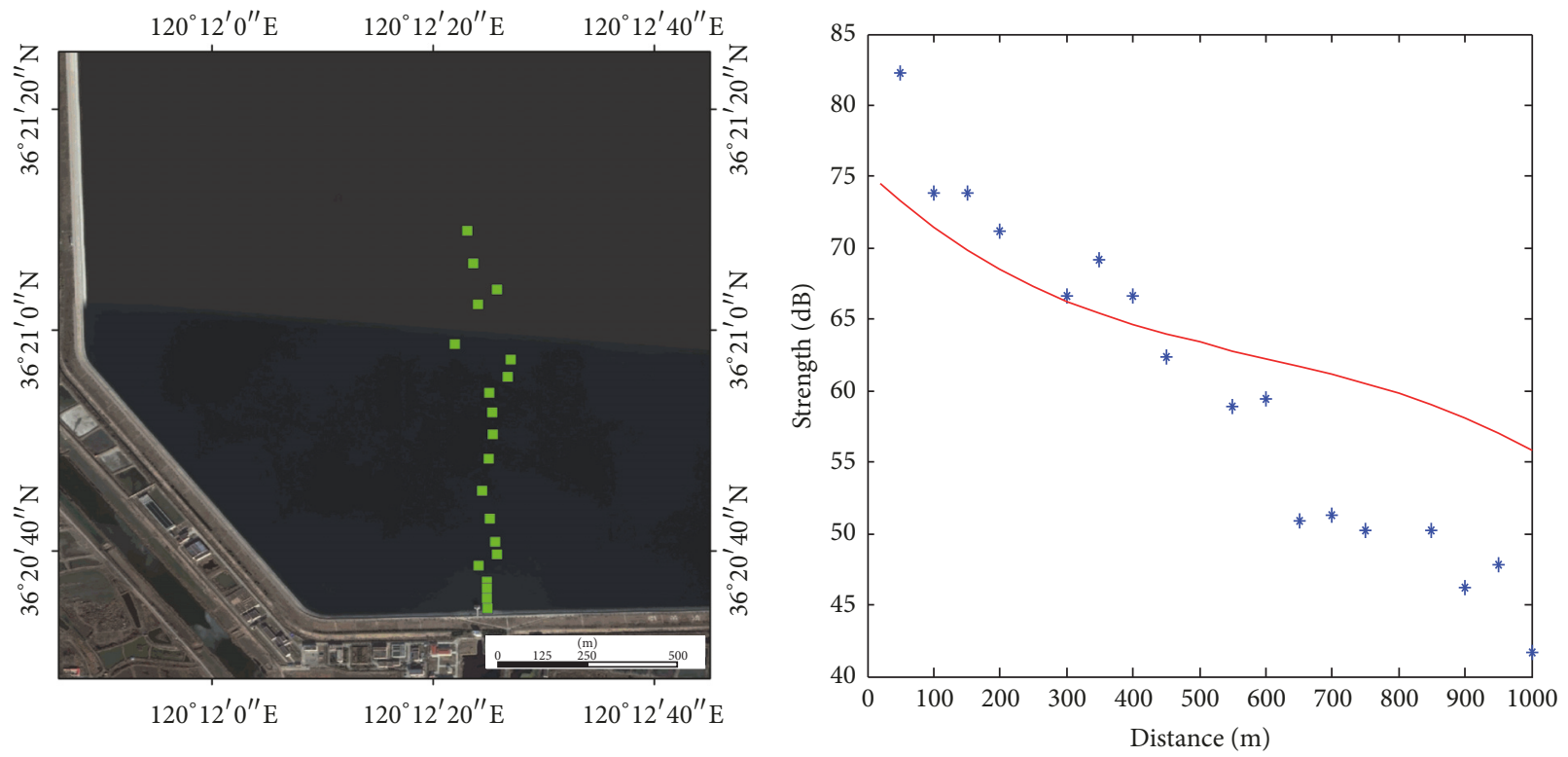

FIGURE 6: Fixed points strength attenuation.

mobile simulation results is about $10 \mathrm{~dB}$, which is caused by the various position of the receiver when the USV is moving.

3.2. Experimental Verification. As shown in Figure 5, we conducted lake experiment using our USV in Qingdao Jihongtan Reservoir on July 15, 2015. The depth is approximately $10 \mathrm{~m}$ and the perimeter of the reservoir is $14.3 \mathrm{~km}$. A self-contained hydrophone with $256 \mathrm{kHz}$ sampling frequency is fixed about one meter under the USV and is used to record and monitor communication signals.

Two EvoLogics underwater communication modems were used in this experiment. One is dipped about $2 \mathrm{~m}$ near the shore platform, while the other is located under the USV as a mobile node which is $3 \mathrm{~m}$ from the water surface. The USV also has other equipment, such as CTD, a weather station, GPS, electronic compass, and single beam depth sounder. The USV automatically reaches the designated location in the straight line route every $50 \mathrm{~m}$. Then, the USV sends and receives signals in stationary status. Due to the tough experimental conditions and improper operation, some data was unfortunately lost at $250 \mathrm{~m}, 500 \mathrm{~m}$, and $800 \mathrm{~m}$.

As shown in Figure 6, the blue star indicates the signal strength and the red curve represents the fitted curve which has been obtained in Figure 3 . Within $1 \mathrm{~km}$, the signal strength attenuates from about $89 \mathrm{~dB}$ to about $42 \mathrm{~dB}$. Compared with the fixed points' simulation results, the trend of the attenuation is the same. After $500 \mathrm{~m}$, the experimental results are larger than the simulation results about $5 \mathrm{~dB}$. We analyze that the main reason is the effect of the wind and waves of the lake.

In order to test the quality evaluation model, we conduct a mobile experiment. We define that the desired trajectory is a straight line along north direction, the starting point is $36^{\circ} 20^{\prime} 35^{\prime \prime} \mathrm{N}, 120^{\circ} 12^{\prime} 25^{\prime \prime} \mathrm{E}$, and the ending point is $36^{\circ} 21^{\prime} 12^{\prime \prime} \mathrm{N}$, $120^{\circ} 12^{\prime} 25^{\prime \prime} \mathrm{E}$. USV sails automatically straight line $1 \mathrm{~km}$ in mobile communication experiment using path following automatic control. The mobile lake experiment results are shown in Figure 7.

As indicated in Figure 7, the blue star indicates the signal strength and the red smooth curve is the mobile fitted curve 

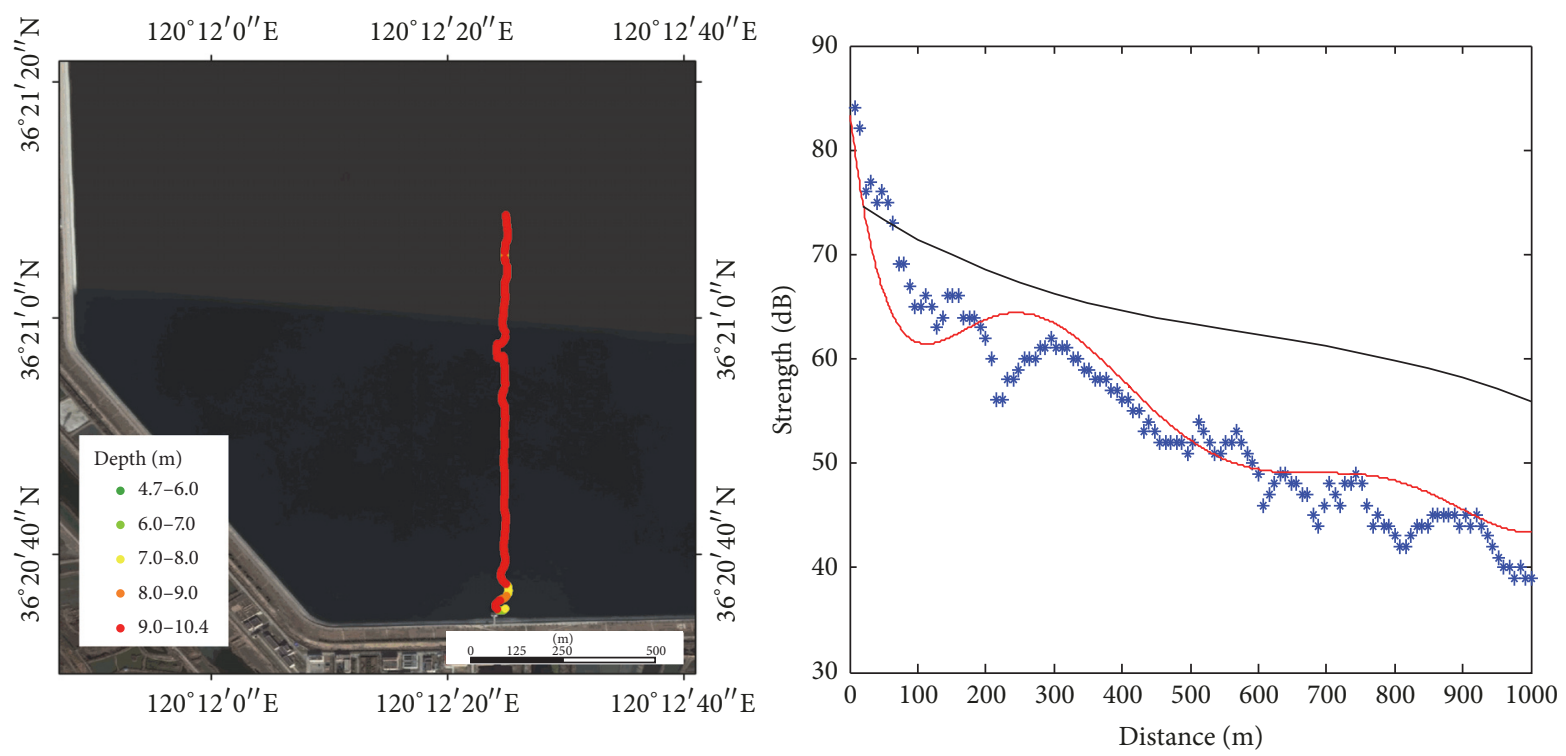

FIGURE 7: Mobile points strength attenuation.

which is shown in Figure 4, and the black curve represents the fixed fitted curve which is depicted in Figure 3. We can see that the attenuating trends of these three curves are consistent in the range. Fixed and mobile experimental results differ about $10 \mathrm{~dB}$, which is caused by the Doppler effect with the movement of the USV.

\section{Conclusions}

In this paper, aiming at some problems such as the difficult construction of UWA communication network and the uncertainty of communication quality, the UWA mobile communication quality evaluation model of the USV is proposed. The simulation and the lake experiments verify the reasonability and the effectiveness of the evaluation model. The model proposed in this paper can not only be used for the network construction of USV UWA nodes, but also provide the basis for constructing the network of other mobile nodes such as the AUV and ROV.

\section{Conflicts of Interest}

The authors declare that they have no conflicts of interest.

\section{Acknowledgments}

The authors gratefully acknowledge the financial support by National Key R\&D Program of China (2017YFC1405203); National Natural Science Foundation of China under Grant 61401111; Public Science and Technology Research Funds Projects of Ocean (201505005-2); National Natural Science Foundation of China (61431005); Fundamental Research Funds for the Central Universities (201213004).

\section{References}

[1] J. Yuh, G. Marani, and D. R. Blidberg, "Applications of marine robotic vehicles," Intelligent Service Robotics, vol. 4, no. 4, pp. 221-231, 2011.

[2] N. A. Cruz, B. M. Ferreira, O. Kebkal et al., "Investigation of underwater acoustic networking enabling the cooperative operation of multiple heterogeneous vehicles," Marine Technology Society Journal, vol. 47, no. 2, pp. 43-58, 2013.

[3] A. Bahr, J. J. Leonard, and A. Martinoli, "Dynamic positioning of beacon vehicles for cooperative underwater navigation," in Proceedings of the 25th IEEE/RSJ International Conference on Robotics and Intelligent Systems (IROS '12), pp. 3760-3767, Vilamoura, Portugal, October 2012.

[4] C. Murphy, J. M. Walls, T. Schneider, R. M. Eustice, M. Stojanovic, and H. Singh, "CAPTURE: a communications architecture for progressive transmission via underwater relays with eavesdropping," IEEE Journal of Oceanic Engineering, vol. 39, no. 1, pp. 120-130, 2014.

[5] Z. Lv, J. Zhang, J. Jin, and L. Liu, "Link strength for Unmanned Surface Vehicle's underwater acoustic communication," in Proceedings of the 2016 IEEE/OES China Ocean Acoustics Symposium, COA 2016, January 2016.

[6] T. C. Yang and S. H. Huang, "Building a database of ocean channel impulse responses for underwater acoustic communication performance evaluation: issues, requirements, methods and results," in Proceedings of the 11th ACM International Conference on Underwater Networks and Systems, WUWNet 2016, October 2016.

[7] J. Senne, A. Song, M. Badiey, and K. B. Smith, "Parabolic equation modeling of high frequency acoustic transmission with an evolving sea surface," The Journal of the Acoustical Society of America, vol. 132, no. 3, pp. 1311-1318, 2012.

[8] A. Radosevic, J. G. Proakis, and M. Stojanovic, "Statistical characterization and capacity of shallow water acoustic channels," in Proceedings of the IEEE Bremen: Balancing Technology with Future Needs (OCEANS '09), pp. 1-8, May 2009. 
[9] B. Borowski, "Characterization of a very shallow water acoustic communication channel," IEEE Xplore, pp. 1-10, 2009.

[10] J. Zhang, J. Cross, and Y. R. Zheng, "Statistical channel modeling of wireless shallow water acoustic communications from experiment data," in Proceedings of the IEEE Military Communications Conference (MILCOM '10), November 2010.

[11] T. K. Sarkar, Z. Ji, K. Kim, A. Medouri, and M. Salazar-Palma, "A survey of various propagation models for mobile communication," IEEE Antennas and Propagation Magazine, vol. 45, no. 3, pp. 51-82, 2003.

[12] P. Qarabaqi and M. Stojanovic, "Statistical characterization and computationally efficient modeling of a class of underwater acoustic communication channels," IEEE Journal of Oceanic Engineering, vol. 38, no. 4, pp. 701-717, 2013.

[13] S.-H. Byun, S.-M. Kim, Y.-K. Lim, and W. Seong, “Time-varying underwater acoustic channel modeling for moving platform," in Proceedings of the Oceans 2007 MTS/IEEE Conference, pp. 1-4, October 2007.

[14] J. Aparicio, F. J. Álvarez, J. Ureña, A. Jiménez, C. Diego, and E. García, "Swell effect in shallow underwater acoustic communications," in Proceedings of the 15th IEEE International Conference on Emerging Technologies and Factory Automation, ETFA 2010, pp. 1-4, September 2010.

[15] N. Jing, W. Bi, and Q. Yue, "Attack simulation model and channel statistics in underwater acoustic sensor networks," Tsinghua Science and Technology, vol. 16, no. 6, pp. 611-621, 2011.

[16] L. Brekhovskikh M and P. Lysanov Y, "Fundamentals of ocean acoustics," in Journal of the Acoustical Society of America, vol. 116, p. 1863, 3rd edition, 1863.

[17] T. I. Fossen, Handbook of Marine Craft Hydrodynamics and Motion Control, John Wiley \& Sons, 2011. 


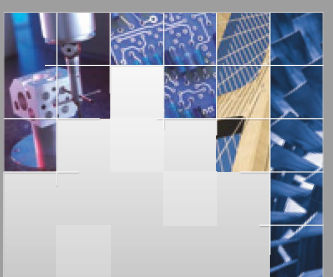

\section{Enfincering}
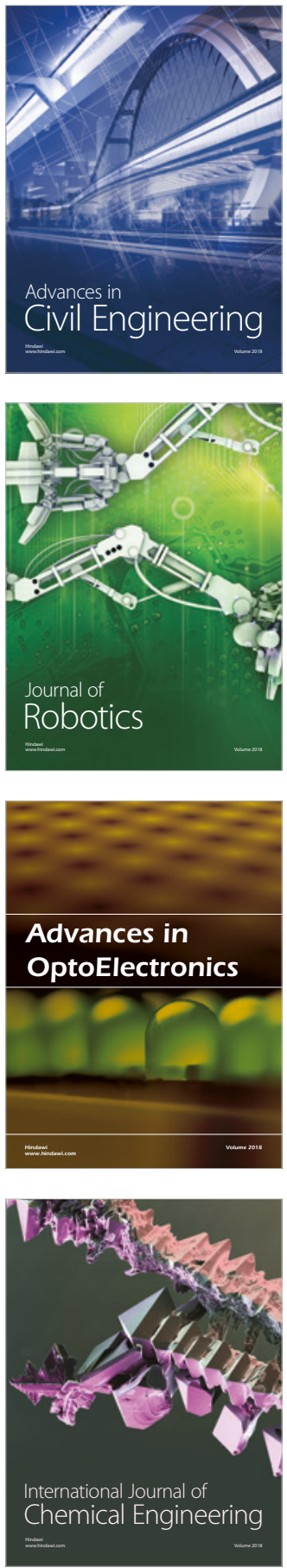

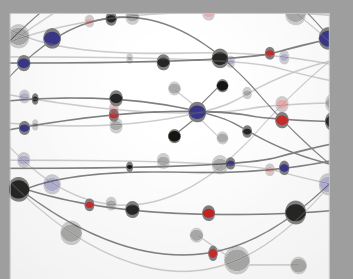

\section{Rotating \\ Machinery}

The Scientific World Journal

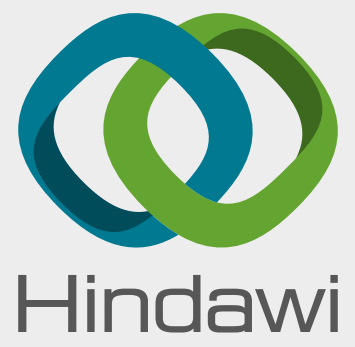

Submit your manuscripts at

www.hindawi.com
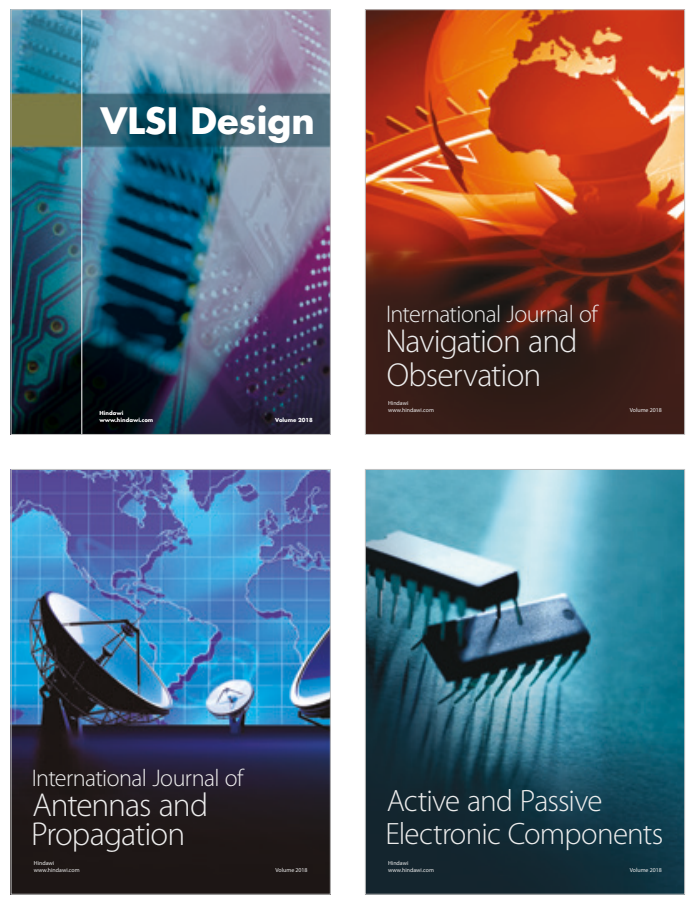
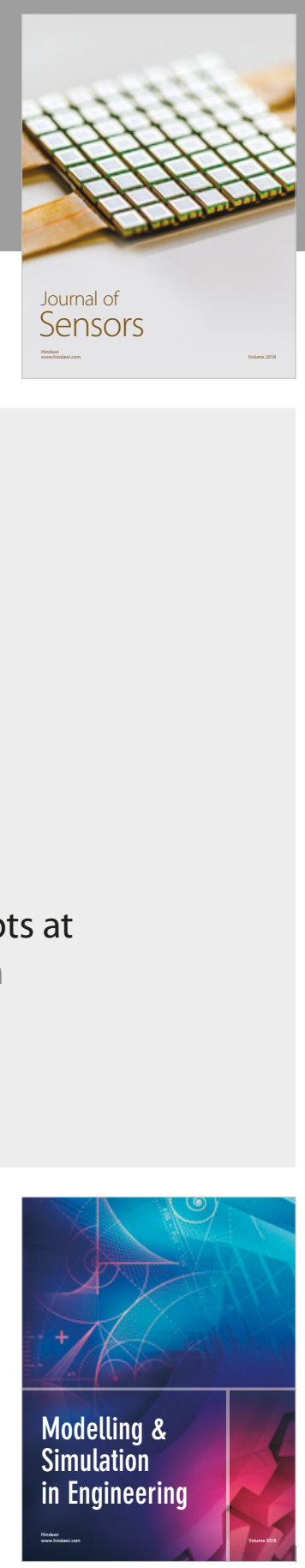

\section{Advances \\ Multimedia}
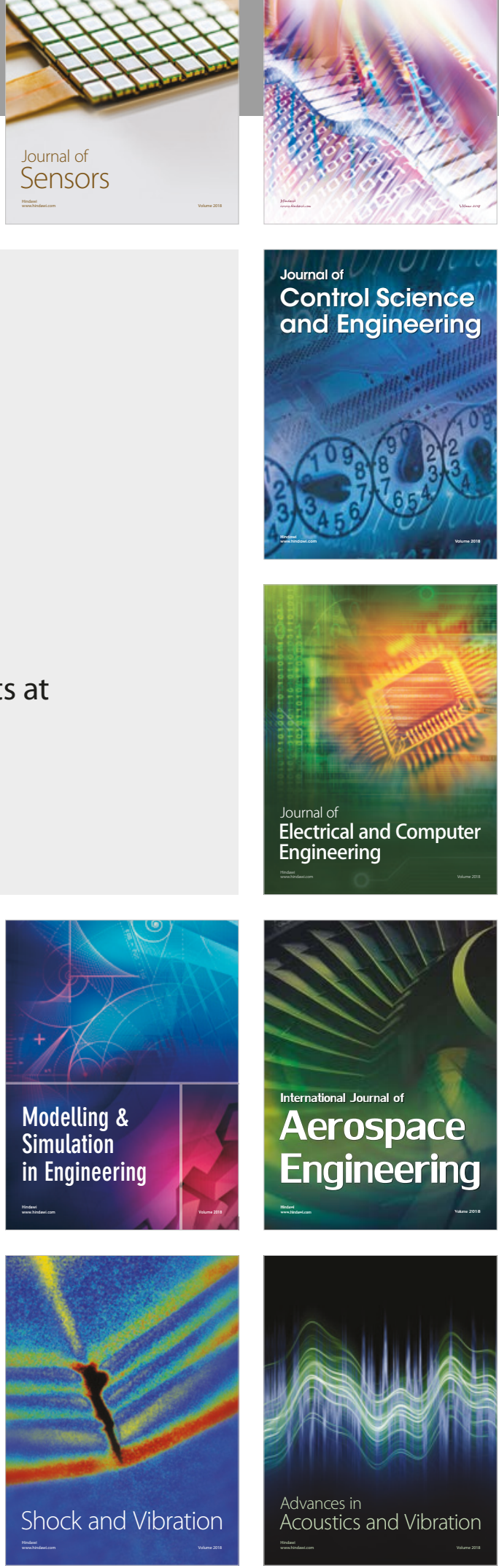